\title{
Estrus detection in dairy cattle: Are vaginal detector probes species-specific to
} vaginal mucus?

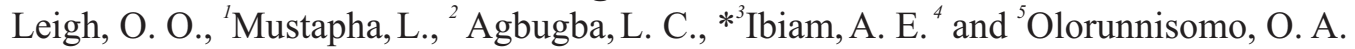
${ }^{1,2,3 \& 4}$ Department of Theriogenology, ${ }^{5}$ Department of Animal Science, University of Ibadan, Ibadan

*Corresponding author: lindaagbugba@gmail.com

Abstract

Two separate estrous detector probes, labelled for bovine and swine, were intuitively used to study changes in electrical resistivity of vaginal mucus (ERVm) in 48 Sokoto Gudali cattle (SGC) that were synchronized with double intramuscular injections of prostaglandin $F_{2}$ alpha (PGF). The cattle comprised of 32 heifers and 16 cows were randomly assigned into groups A (11-day) and B (12-day) PGF injection intervals, with equal number of heifers and cows. The ERVm was measured between first day (d 0) of PGF injection and d 15. Each injection contained $500 \mu \mathrm{g}$ Estrumate ${ }^{\circledR}$. Results showed that the difference in the mean values of ERVm between bovine (267.07 220.81$)$ and swine (256.64 139.41$)$ probes was not significant $(p>0.05)$. Similarly, the differences in ERVm between the bovine and swine probes at $48 \mathrm{~h}$ post $1^{\text {st }} \mathrm{PGF}$ in all cattle (239.20 \pm 5.09 and $\left.231.60 \pm 13.53\right)$, as well as $48 \mathrm{~h}$ post

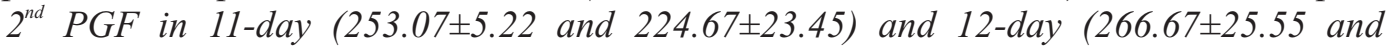
$233.03 \pm 21.96) P G F$ injection groups were not significant ( $p>0.05)$, respectively. In all, $75 \%$ of cattle stood to mounting by pen mates and a 75:25 success to failure ratio for evaluating ERVm in cattle with the aid of a swine vaginal probe were obtained. The ERVm pattern also suggests that estrus occurred between 0-72 h post PGF with both probes. It can be concluded that there may be no difference in sensitivity to vaginal mucus between these two probes and that either may be used to evaluate ERVm in the SGC.

Keywords: Estrus detection, vaginal mucus resistivity, probes, Sokoto Gudali cows

\section{Introduction}

The delicate sequence between production and reproduction cycles is an indicator of performance efficiency in dairy industries. Some of the factors known to threaten this sequence of events include disease factors, poor genetics, malnutrition and poor reproductive management (Hudson et al., 2012). According to Marinho et al. (2013), classical indicators of optimal reproduction in the dairy industry have nose-dived over the last 50 years or more. Some of these indicators include the rates of conception at first service and services per conception as well as the calving interval. One parameter that is central to all these indicators and also a component of good reproductive management programs in all species of livestock is estrus detection accuracy which is the percentage of estrus periods observed that are actual estrus periods (Nebel, 1988). Error rates in estrus detection ranging from 0-60\% (Reimers et al., 1985), 1-12\% (Elmore, 1989), and 25\% (Nebel et al., 1987), have been reported among cows presented for insemination. These figures must have in no small measure contributed to grave losses in the industry. According to Williamson and Morris (1972), among the signs of estrus such as grouping, ruffled tail head, homosexual activity, standing immobile to mounting was the most consistent in $79 \%$ of cows. The problems associated with estrus detection in cattle are however, multifactorial. Apart from the brevity of its duration (Trimberger, 1948), many of the indicators are unreliable and inconsistent, evaluation techniques are 


\section{Non-species-specificity of vaginal probes with mucus.}

laborious, leading to poor estrus detection and pregnancy rates. For example, the best indicator of estrus being standing immobile to mounting (Gwasdauskas et al., 1986), visual observation of this behavior will be the most productive way for efficient estrus detection. However, about $57 \%$ and $43 \%$ of cows show such signs between 6 am midnight and midnight -6 am, respectively. This implies that a 24-hourly observation would be required to obtain $100 \%$ detection rate. The reality of the challenges involved in estrus detection have therefore led to the development of estrus detection aids (EDA), some of these include: vaginal temperature monitoring (Brehme et al., 2008), progesterone testing (Claycomb and Delwiche, 1998), pedometers (Rodenberg and Murray, 2007), altered vocalization (Schon et al., 2007), heat watch (Heatwatch, 1992), and pressure sensing device (Dransfield, 1998). Several authors (Palmer et al., 2010; Johnson et al., 2012; Kamphius et al., 2012; Talukdaer et al., 2015) have attested to the sensitivity and positive predictive values of these aids in estrus detection. Perhaps, a more recent addition to existing EDA in Nigeria is the estrous detector probes which measure electrical resistivity of vaginal mucus $(E R V m)$. The dynamic nature of the biophysical and biochemical characteristics of cervico-vaginal/vaginal mucus during the estrous cycle in cows has been established (Carlstedt and Sheehan, 1989; Rangnekar et al., 2002). These changes are influenced by the endocrinological status of the reproduction cycle and detected as fluctuations in ERVm during different stages of the cycle (LopezGatius et al., 1993). According to some reports, changes in ERVm have offered usefulness in determining breeding time in the ewe (Theodosiadou and Tsiligianni, 2015), cattle (Heckman et al., 1979; Tsiligianni et al., 2000; Bernardi et al.,
2016), buffaloes (Verma et al., 2014) and women (Pommerenke, 1946). Estrous detector probes are manufactured in different sizes and lengths according to species of animals and are readily available in the market. In this report, we present our recent observations arising from an intuitive application of two estrous detector probes (bovine and swine), while monitoring electrical resistivity of the vagina mucus in Sokoto Gudali cattle treated with prostaglandin $\mathrm{F}_{2}$ alpha.

\section{Materials and methods Study site}

The study was carried out at the Dairy Unit of the Teaching and Research Farm, University of Ibadan, Oyo State, Southwest Nigeria. Ibadan city lies on latitude $7^{\circ} 27^{\prime}$ and longitude $3^{\circ} 45^{\prime}$. It has an average annual rainfall of about $1250 \mathrm{~mm}$ with mean temperature of $27^{\circ} \mathrm{C}$.

\section{Study animals}

Forty-eight Sokoto Gudali cattle consisting of 32 heifers $(175-240 \mathrm{~kg})$, that have cycled at least once) and 16 cows (1-3 parity, about 60 days post calving) were used for the study. The animals had ear tags for identification and were kept in their pens throughout the day except when they grazed within the paddock to allow cleaners work in the pens. The cows were fed with silage and chopped elephant grass. Clean water was also provided ad libitum.

\section{Study design}

Cattle were randomly assigned into groups $A$ and $B$ such that each group had 16 heifers and 8 cows.

\section{Estrus synchronization and identification of estrus}

The double prostaglandin $\mathrm{F}_{2}$ alpha (PGF) protocol was used. Cows in Groups A and B were administered (i/m) $500 \mu \mathrm{g}$ of Estrumate $^{\circledR}$ (Schering-Plough Animal Health Corp, Germany) at 11- and 12-day intervals, respectively. An animal was 


\section{Leigh, Mustapha, Agbugba, Ibiam and Olorunnisomo}

regarded as being in estrus only if she accepted mounting by standing still to another animal for at least 1 minute. Arbitrary mountings that were sustained for less than a minute were disregarded. Animals were watched for mountings thrice (7 am, 11 am and $5 \mathrm{pm}$ ) daily, each time for two hours from the point of $2^{\text {nd }}$ PGF administration until 96 hours later.

Evaluation of electrical resistivity of vaginal mucus (ERVm)

Draminski estrous detector probes for bovine and swine were used. The procedure described by Kitwood et al. (1993) was slightly modified. Values of ERVm were read thrice at 8.00am and the average taken as the daily reading per animal, starting from the day of first administration of PGF (d 0) in both groups until 96 hours after PGF treatment in group A (d 15). The reading could not be taken on the second day (d 2) due to a farm operation.

Evaluation of graphs of electrical resistivity of vaginal mucus (ERVm) obtained with bovine and swine probes

Line graphs were plotted with ERVm on the ordinate and days on the abscissa. The criteria used for comparison of graphs (Figures) obtained were as follows:

S i milarity/Dissimilarity of appearance/pattern of graphs between the bovine and swine probes during 0-96 hours after administration of the $2^{\text {nd }}$ PGF in both Groups of cattle.

Similarity connoted patterns of ERVm in which the two probes either showed a spike or a decline, while dissimilarity connotes that either showed a spike and the other a decline or plateau during 0-96 h post PGF treatment.

\section{Statistical analysis}

Data were analysed using descriptive statistics and the student t-test at 95\% confidence interval (Elston and Johnson, 2008). Values of $p<0.05$ were considered significant.

\section{Guide on research conduct}

The study was conducted under strict adherence to the principles of the care and use of farm animals in research, teaching and testing of the Canadian Council on Animal Use (CCAC, 1993).

\section{Results}

Comparison of electrical resistivity of vaginal mucus

Table 1 shows the differences in electrical resistivity of vaginal mucus (ERVm) obtained with bovine and swine probes. For the 48 cattle, the difference in the mean values of ERVm between bovine $(267.07 \pm 20.81)$ and swine $(256.64 \pm 39.41)$ probes during the study was not significant $(p>0.05)$. Similarly, the differences between values of ERVm by the bovine and swine probes at $48 \mathrm{~h}$ post $1^{\text {st }}$ PGF in all cows (239.20 \pm 5.09 and $231.60 \pm 13.53)$ and at 48 h post $2^{\text {nd }} P G F$ for 11-day $(253.07 \pm 5.22$ and $224.67 \pm 23.45)$ as well as 12-day (266.67 \pm 25.55 and $233.03 \pm 21.96)$ PGF interval groups were not significant ( $p>0.05)$, respectively.

Table 1: Comparison of electrical resistivity of vaginal mucus obtained with Draminski bovine and swine estrous detector probes in cattle treated with double injections of prostaglandin $F_{2}$ alpha (Estrumate ${ }^{\circledR}$ )

\begin{tabular}{llll}
\hline Criteria & $\begin{array}{l}\text { Bovine probe } \\
(\text { Mean } \pm \text { S.D) }\end{array}$ & $\begin{array}{l}\text { Swine probe } \\
(\text { Mean } \pm \text { S.D) }\end{array}$ & $\begin{array}{l}\text { P-value } \\
\text { /Significance }\end{array}$ \\
\hline Readings; d $(0-15)$ & $267.07 \pm 20.81$ & $256.64 \pm 39.41$ & $0.223 / \mathrm{p}>0.05$ \\
48 h post ${ }^{\text {st }}$ PGF (in all cattle) & $239.20 \pm 5.09$ & $231.60 \pm 13.53$ & $0.667 / \mathrm{p}>0.05$ \\
48 h post 2 ${ }^{\text {nd }}$ PGF $(11-d$ treatment cattle) & $253.07 \pm 5.22$ & $224.67 \pm 23.45$ & $0.131 / \mathrm{p}>0.05$ \\
48 h post 2 ${ }^{\text {nd }}$ PGF $(12-d$ treatment cattle) & $266.67 \pm 25.55$ & $233.03 \pm 21.96$ & $0.154 / \mathrm{p}>0.05$ \\
\hline
\end{tabular}


Non-species-specificity of vaginal probes with mucus.

Relationships in the patterns of electrical resistivity of vaginal mucus (ERVm)

Figure 1 shows that $25 \%$ of cows showed dissimilarity in their patterns of ERVm while $58 \%$ had similar pattern and $17 \%$ had a different but also similar pattern of ERVm.

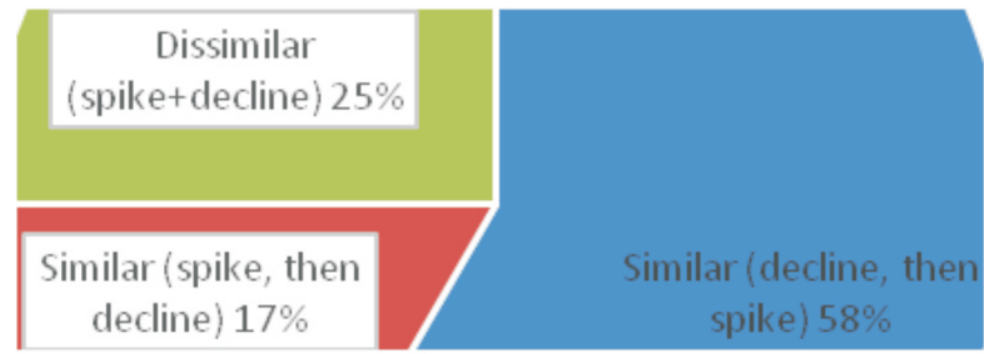

Figure 1: Relationships in the patterns of electrical resistivity of vaginal mucus (ERVm) obtained with Draminski bovine and swine estrous detector probes in cattle at 0-96 h post double injections of prostaglandin $F_{2}$ alpha (Estrumate ${ }^{\circledR}$ ).

\section{Graphs of ERVm in cows}

Figure 2 shows the dissimilar pattern in values of ERVm. Within the $25 \%$ that showed dissimilar pattern of ERVm as in Figure 1, the bovine probe showed a continued increase in ERVm up to $24 \mathrm{~h}$ after PGF treatment but decreases slowly thereafter for about $48 \mathrm{~h}$ and then peaks to the end of the study (i.e. 96 h). During the same periods, the pattern of the swine probe was exactly opposite.

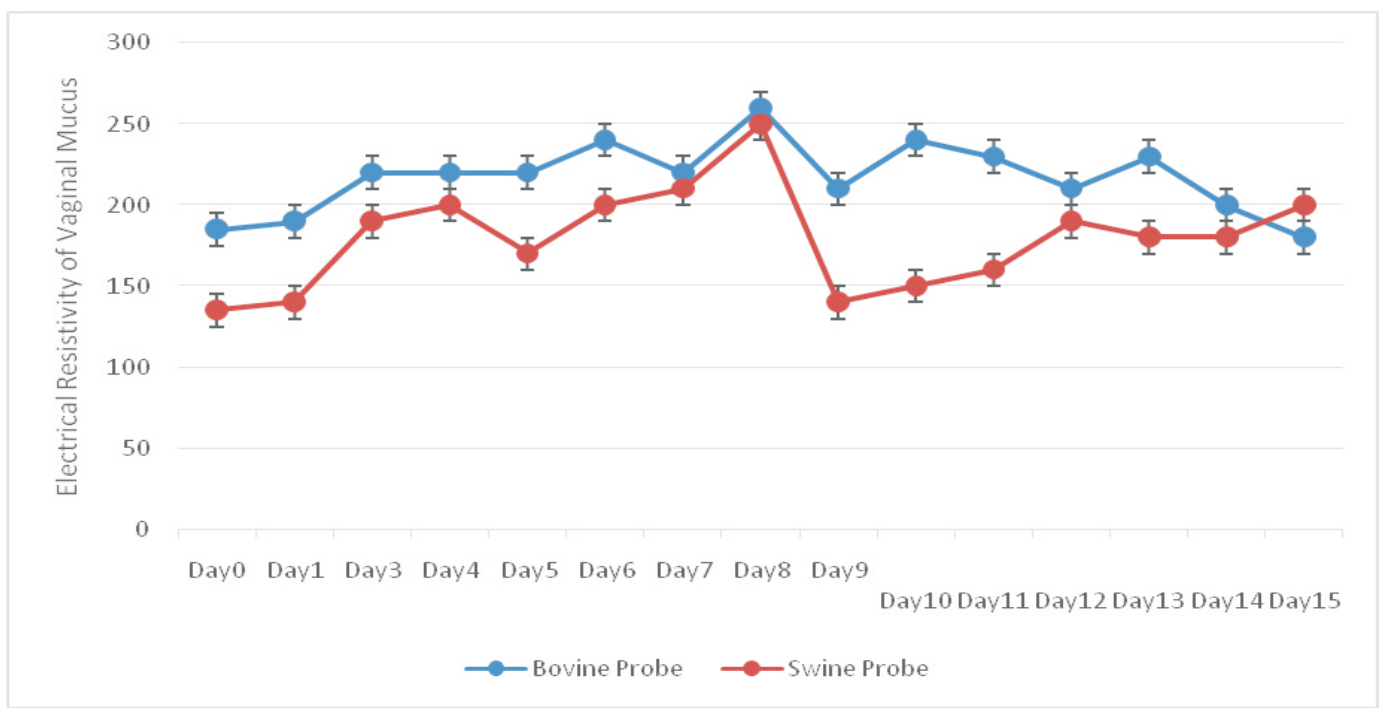

Figure 2: Line graph of ERVm in cattle showing dissimilarity between bovine and swine estrous detector probes during 0-96 h post $2^{\text {nd }}$ PGF (i.e. d 11-15). 


\section{Leigh, Mustapha, Agbugba, Ibiam and Olorunnisomo}

Figure 3 shows a similar pattern with spike during $0-48 \mathrm{~h}$ post $2^{\text {nd }} \mathrm{PGF}$ although, that of the swine probe was for $0-24 \mathrm{~h}$, and a decline thereafter with both probes up to 72 $h$ and a slight peak to the end of the study. Figure 4 shows another similar pattern between the two probes. The values of ERVm with bovine probe started to decline during $0-48 \mathrm{~h}$ post $2^{\text {nd }}$ PGF while that for swine declined from $24 \mathrm{~h}$ post treatment. Both spiked from $48 \mathrm{~h}$ post treatment to the end of the study.

Figures 3 and 4 also represent the pattern for the cows that accepted mounting by pen mates. In Figure 3, the pattern of ERVm suggests that estrus occurred between 24$72 \mathrm{~h}$ post PGF (swine probe), and $48-72 \mathrm{~h}$ (bovine probe). In Figure 4, the pattern of ERVm suggests that estrus occurred between 24-48 h post PGF (swine probe), and $0-48 \mathrm{~h}$ (bovine probe).

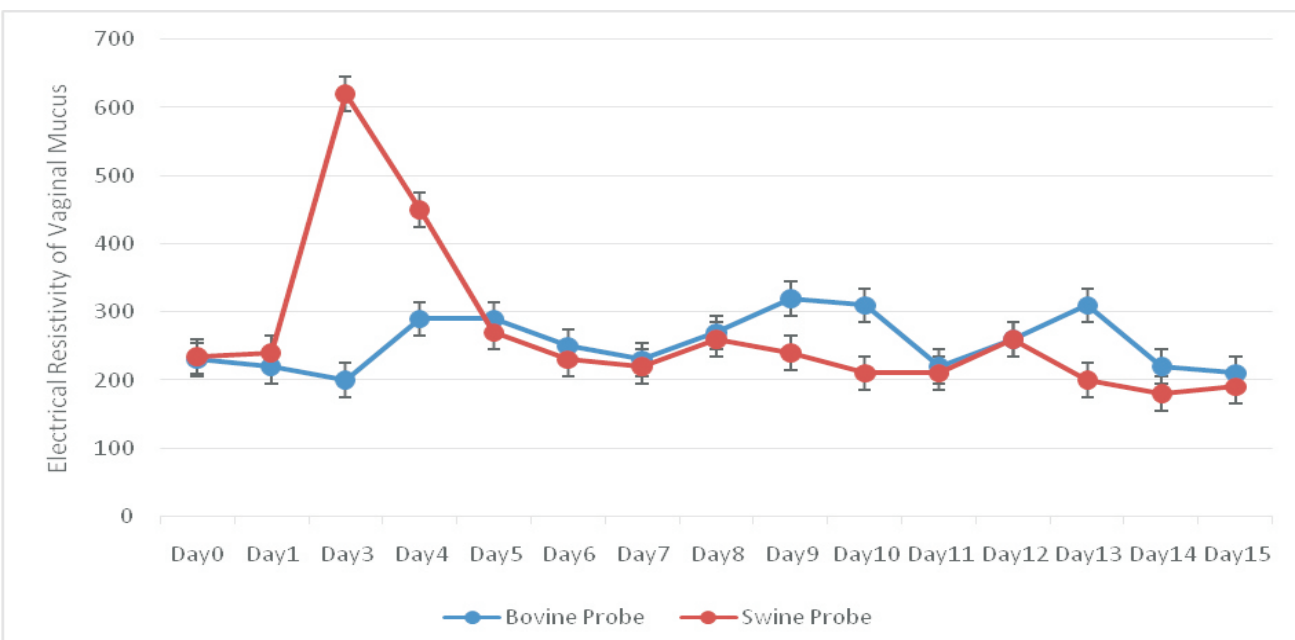

Figure 3: Line graph of ERVm in cows showing similarity (decline) between bovine and swine estrous detector probes during 0-96 h post $2^{\text {nd }}$ PGF (i.e.d 11-15).

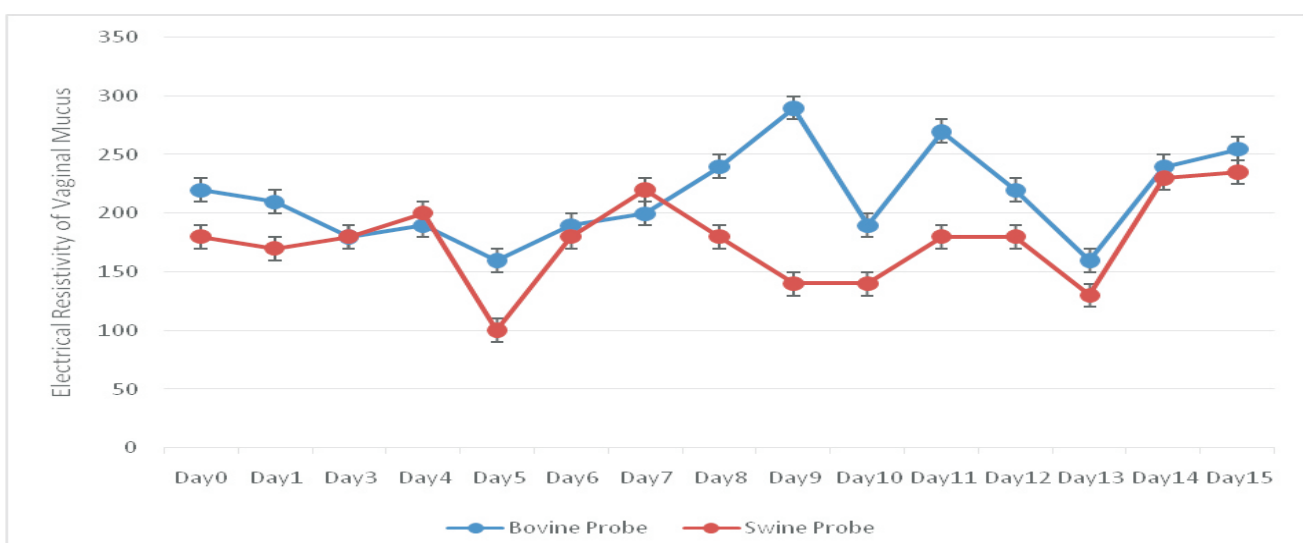

Figure 4: Line graph of ERVm in cows showing similarity between bovine and swine estrous detector probes during 0-96 $\mathrm{h}$ post $2^{\text {nd }}$ PGF (i.e. d 11-15). 


\section{Non-species-specificity of vaginal probes with mucus.}

\section{Discussion}

Since values of electrical resistivity of vaginal mucus (ERVm) reflect the reproductive endocrinological milieu of the cows species at any given time (Roth, 1993), it may be expected that readings evaluated with a species-specific probe would provide information that are specific for the species and distinct from readings obtained using other probes that are not labelled for same species. Our results however showed non-significant differences between the values of ERVm obtained using bovine and swine probes in cattle. Simply, there may be non-significant between the probes that may warrant species-specific labelling as they are usually marketed. This would imply that outside of the differences in size and length of the shaft (bovine being longer than swine) inserted into the vagina, the sensitivities of the equipment are comparable. This ought not to be, considering the clinical value of ERVm as a useful estrus detection aid. Figure 1 appears to buttress the position suggested by the earlier finding in this study as it showed that $75 \%$ of the patterns of ERVm obtained by both probes were similar. Although among this proportion, $17 \%$ and $58 \%$ of ERVm from the animals as evaluated by both probes agreed separately in pattern. The proportion of the ERVm pattern that was dissimilar may be viewed in a different perspective. A consideration of the line plot for the bovine probe in this group (Figure 2) of cattle closely resembles the pattern obtained in Figure 3 where evidence of similarity between both probes was found. It therefore becomes obvious that $25+17 \%$ i.e. $42 \%$ of cows would have a similar pattern of ERVm (Figure 3) and another $58 \%$ cows would have another similar (Figure 4), but different from the earlier pattern of ERVm. Minor differences among individual cows e.g. 11- and 12-day PGF treatment interval may be responsible for the similar but different groups into which the cows would then be categorized. Be that as it may, it would still subsist that there is a 75:25 success to failure ratio for evaluating ERVm values in cows with the aid of a swine vaginal probe. This success rate may be viewed as high enough, making species labelling unnecessary, except for probe length. In the current study, two injections of prostaglandin $\mathrm{F}_{2}$ alpha (PGF) were administered to cycling cows, implying that 75 to $85 \%$ at least should exhibit estrus within two (48 hours) to four (96 hours) days after the second injection (Day, 2005). Our observation on the proportion of cows that stood to mounting by pen mates is in agreement with this earlier report as $75 \%$ of the cows were in heat. The actual figure in heat may be more than this figure since deficits can occur due to unobserved estrus. Among the animals that showed standing estrus, the pattern of ERVm further suggest that in all cases, estrus occurred between 0$72 \mathrm{~h}$ post PGF treatment with both probes. This result is in agreement with several studies (Voh Jr. et al., 2000; Venkata et al., 2013) and further validate the instructional guide attached to the equipment that estrus is always characterized by a decline, preceded by a spike in ERVm. The agreement between the two probes in detecting estrus is also worrisome, lending credence to the probable similarity in the sensitivities of both probes and possibly raising a query on the clinical advantage of species labelling and electrical resistivity of vaginal mucus. This finding may suffice to suggest that the different probes interacted in a similar way with the vaginal mucus produced at the different stages of the study. Apart from the fact that obvious differences with respect to reproductive anatomy exist between the swine and the bovine, salient differences also exist in their reproductive endocrinology e.g. their lengths of estrus, 


\section{Leigh, Mustapha, Agbugba, Ibiam and Olorunnisomo}

duration of ovulation and luteal phases as well as vaginal cytology, even though, lengths of the cycle are similar. If these differences are peculiar enough, they are likely to affect the nature of the vaginal mucus and sufficient therefore to be factored into the manufacture of a speciesspecific vaginal probe. Some reports have shown that hormonal treatments in animals for estrus induction often disrupt the physico-chemical nature of cervicovaginal mucus (Tsiligianni et al., 2001). It is not unlikely that a similar effect as this is seen in the present study or how else would it be explained that a vaginal probe labeled for swine produced comparable values ( $p>0.05)$ of ERVm to the one specific for bovine in cattle $\square$ These findings can only strengthen earlier implication that a great similarity is in both the bovine and swine probes, suggesting that in cattle, either probe may be applicable. The present authors strongly feel that species peculiarities in terms of physico-chemical properties of vaginal mucus, except they are identical across species or the ones in question, ought to distinguish between values obtained with different probes in a single species to warrant labelling as such. Otherwise, it will be scientific and appreciative of the physiological differences between the swine and the bovine not to have reduced the differences between these species in terms of the biology of the vaginal mucus, if there are any, to difference in the sizes of the probes as our findings in this study suggest.

\section{Conclusion}

The study showed that there may be no species-sensitivity differences between the Draminski bovine and swine probes and that either may be used to evaluate electrical resistivity of vaginal mucus in Sokoto Gudali cattle treated with prostaglandin $\mathrm{F}_{2}$ alpha.

\section{Acknowledgement}

The authors gratefully acknowledge the assistance of Miss Labake Bello of the Department of Animal Science, University of Ibadan, during the stage of data collection.

\section{References}

Bernardi, S., Rinaudo, A. and Marini, P. 2016 . Cervical mucus characteristics and hormonal status at insemination of Holstein cows. Iran J. Vet. Res. 17(1), 45-49.

Brehme, U., Stollberg, U., Holz, R. and Schleusener, T. 2008. ALT pedometer-New sensor aided measurement system for improvement in oestrus detection. Comput. Electron. Agric. 62, 73-80.

Canadian Council on Animal Care Guide ( C C A C ) . 1993 . http://www.ccac.ca/Documents/ Standards/Guidelines/Experiment al_Animals Vol1.pdf; [Accessed 4 November 2015].

Carlstedt, I. and Sheehan, J. K. 1989. Structure and macromolecular properties of cervical mucus glycoproeitns. In: Mucus and Related Topics. E. Chantler and N.A. Ratcliffe (Eds). Society for Experimental Biology, Cambridge University Press (UK), pp 289-316.

Claycomb, R. W. and Delwiche, M. J. 1998. Biosensor for on-line measurement of bovine progesterone during milking. $J$. Dairy Sci. 81, 2890-2896.

Day, M. L. 2005. Systems of synchronization with prostaglandin $\mathrm{F}_{2}$ alpha. In: Handbook of estrous synchronization. Western region Publication No. 014. Ohio Agricultural Research and Development Center. The Ohio State University, 1680 Madison 


\section{Non-species-specificity of vaginal probes with mucus.}

Avenue, Wooster, Ohio 44691.

Dransfield, M. B. G., Nebel, R. L., Pearson, R. E. and Warnick, L. D. 1998. Timing of insemination for dairy cows identified in estrus by a telemetric estrus detection system. J. Dairy Sci. 81, 18741882.

Elmore, R. G. 1989. The use of rapid progesterone assays in dairy practice. Agri-Pract. 10, 5.

Elston, R. C. and Johnson, W. D. 2008. Basic Biostatistics for Geneticists and Epidemiologists: A Practical Approach. John Willey and Sons Ltd., UK. pp 233-246.

Gwazdauskas, F. C., Whittier, W. D., Vinson, W. E. and Pearson, R. E. 1986. Evaluation of reproductive efficiency of dairy cattle with emphasis on timing of breeding. $J$. Dairy Sci. 69, 290.

Heat Watch User's Manual. ABS Global, De Forest. 1992., WI. P. 4.5, 4.8.

Heckman, G. S., Katz, L. S., Foote, R. H., Oltenacu, E. A. B., Scott, N. R. and Marshall, R. A. 1979. Estrous cycle patterns in cattle monitored by electrical resistance and milk progesterone. J. Dairy Sci. 62, 6468.

Hudson, C., Kerby, M., Statham, J. O. and Wapenaar, W. 2012. Managing Herd Health. In: Dairy Herd Health. M Green (Eds). CABI International (UK), pp 7380.

Johnson, C. R., Ayers, M. W., Ahmadzadeh, A., Shafii, B., Etter, S., Chebel, R. C. and Dalton, J. C. 2012. Short communication: Characterization of early postpartum estrous behavior and ovulation in lactating dairy cows using radiotelemetry. $J$. Dairy Sci. 95, 5085-5088.

Kamphuis, C., DelaRue, B., Burke, C. R. and Jago, J. 2012. Field evaluation of 2 collar-mounted activity meters for detecting cows in estrus on a large pasture- grazed dairy farm. J. Dairy Sci. 95, 30453056.

Kitwood, S. E., Phillips, C. J. C. and Weise, M. 1993. Use of a vaginal mucus impedance meter to detect estrus in the cow. Theriogenology. 40(3), 559-569.

Lopez-Gatius, F., Miro, J., Sebastian, I., Ibarz, A. and Labernia, J., 1993. Rheological properties of the anterior vaginal fluid from superovulated dairy heifers at estrus. Theriogenology. 40, 167-180.

Marinho, L. S. R., Machado, F. Z. and Seneda, M. M. 2013. Strategies to improve the reproductive efficiency of dairy cattle. In: Dairy Cows. C.T. Hernandez (Eds). Nova Science Publishers Inc., Londrina PR (Brazil), pp 16-22.

Nebel, R. L. 1988. On-farm milk progesterone tests. J. Dairy Sci. 71, 1682.

Nebel, R. L., Whittier, W. D., Cassel, B. G. and Britt, J. H. 1987. Comparison of on-farm and laboratory milk progesterone assays for identifying errors in detection of estrus and diagnosis of pregnancy. J. Dairy Sci. 70, 1471.

Palmer, M. A., Olmos, G., Boyle, L. A. and Mee, J. F. 2010. Estrus detection and estrus characteristics in housed and pastured HolsteinFriesian cows. Theriogenology. 74, 255-264.

Pommerenke, W. T. 1946. Cyclic changes in the physical and chemical properties of cervical mucus. Am. J. Obstet. Gynecol., 52, 1023-9.

Rangnekar, M.N., Dhoble, R.L., Gacche, M.G., Ingawale, M.V., Sawale, A.G., Jadhav, J.M., 2002. 
Physical properties of oestrual cervical mucus in repeat breeding crossbred (Holstein Friesian) cows with reference to fertility. Ind. $J$. Anim. Sci. 72(12), 1122-1124.

Reimers, T. J., Smith, R. D. and Newman, S. K. 1985. Management factors affecting reproductive performance of dairy cows in the northeastern United States. J. Dairy Sci. 68, 963.

Rodenburg, J. and Murray, B. 2007. Pedometry to Improve Reproduction. Order No. 07- 071 AGDEX 410/20.

Roth, B. 1993. Fertility awareness as a component of sexuality education. Preliminary research findings with adolescents. Nurse Pract. 18, 40-48.

Schon, P. C., Hamel, K., Puppe, B., Tuchscherer, A., Kanitz, W. and Manteuffel, G., 2007. Altered Vocalization Rate during the estrus Cycle in Dairy Cattle. J. Dairy Sci. 90, 202-206.

Talukder, S., Thomson, P. C., Kerrisk, K. L., Clark, C. E. F. and Celi, P. 2015. Evaluation of infrared thermography body temperature and collar-mounted accelerometer and acoustic technology for predicting time of ovulation of cows in a pasture-based system. Theriogenology. 83, 739-748.

Theodosiadou, E. and Tsiligianni, T. 2015. Determination of the proper time for mating after oestrous synchronisation during anoestrous or oestrous by measuring electrical resistance of cervical mucus in ewes. Vet. Med. (Praha)., 60(2), 87-93.

Trimberger, G. H. 1948. Breeding efficiency in dairy cattle from artificial insemination at various intervals before and after ovulation. Nebraska Agric. Exp. Stn. Res. Bull, 153,1.

Tsiligianni, T. H., Karagiannidis, A., Brikas, P., and Saratsis, P. H. 2000. Relationship between certain physical properties of cervical mucus and fertility in cows. Deutsche Tierärzt. Wochensch., 106, 28-31.

Tsiligianni, T.H., Karagiannidis, A., Brikas, P., Saratsis, P.H. 2001. Physical properties of bovine cervical mucus during normal and induced by progesterone and/or $\mathrm{PGF}_{2}$ alpha estrus. Theriogenology. 55, 629-640.

Venkata, R.K., Rao, K.S., Supriya, K. and Rajanna, N. 2013. Effect of prostaglandin on estrus response and conception rate in lactating ongole cow. Vet. World. 6(7), 413415.

Verma, K.K., Prasad, S., Kumaresan, A., Mohanty, T.K., Layek, S.S., Patbandha, T.K. and Chand, S. 2014. Characterization of physicochemical properties of cervical mucus in relation to parity and conception rate in Murrah buffaloes. Vet. World. 7(7), 467471.

Voh Jr., A. A., Ogwu, D. and Oyedipe, E. O. 2000. Determination of optimum fixed time artificial insemination of zebu cows following synchronization of estrus with prostaglandin $\mathrm{F}_{2}$ alpha. Nig. Vet. J. 21, 38-50.

Williamson, N. B. and Morris, R. S. 1972. A study of estrous behavior and estrous detection methods in a large commercial dairy herd. Vet. Res. 91,50 .

Received: $10^{\text {th }}$ September, 2018 Accepted: $21^{\text {st }}$ December, 2018 